\title{
Design of a Metamaterial Cavity for Harmonic Terahertz Gyrotrons
}

\author{
Zi-Wen Zhang, Chao-Hai Du*, Senior Member, IEEE, Fan-Hong Li, Juan-Feng Zhu, and Pu-Kun Liu, \\ Senior Member, IEEE, Liang Zhang, Wenlong He, HuaBi Yin, Kevin Ronald, Alan D.R. Phelps, Adrian \\ W. Cross
}

\begin{abstract}
A resonant cavity is developed with a metallic photonic crystal (MPC) to reduce the magnetic field strength and suppress mode competition for a terahertz fourth-harmonic gyrotron. Due to the photonic band gap, electromagnetic waves radiated in certain directions at specified frequencies cannot propagate in the photonic crystal. The dispersion relations of MPC were numerically calculated using a finite-difference frequency-domain method. The gap map for a square lattice of metallic rods was derived from the dispersion relations as the radius of the rods varies. The map of the localized modes in the defects was plotted to illustrate the connection between the band structure and the TE modes. The MPC cavity was simulated and better performance was demonstrated in terms of mode competition compared with a circular waveguide cavity of equivalent cut-off radius at $1-\mathrm{THz}$.
\end{abstract}

Keywords-harmonic operation, photonic crystal, photonic band gap, mode competition, gyrotron

\section{INTRODUCTION}

High-power electromagnetic sources in the terahertz $(\mathrm{THz})$ band have attracted extensive interest for its potential applications in fields of communication, radar, environmental monitoring, spectroscopy, etc. But current commercial devices do not meet the requirements of both high power and broad bandwidth [1]-[4]. The gyrotron is an vacuum electronic device based on the electron cyclotron resonance maser instability and is capable of generating high-power electromagnetic radiation in the millimeter and $\mathrm{THz}$ band. As the frequency increases to the $\mathrm{THz}$ band, researchers are aiming for higher operating efficiency for high-order harmonic gyrotron operation [4].

In 1987, Professor Yablonoviteh of Bell Labs and Professor John of Princeton University proposed the concept of photonic crystals (PC) independently [5]-[6]. Due to photonic band gap (PBG), a photonic crystal can offer complete control over electromagnetic wave (EMW) propagation, especially preventing the EMW from propagating in certain directions with specified frequencies [7]. This provides theoretical support for changing the fundamental operating state of gyrotrons. In 2001 a gyrotron amplifier at $140-\mathrm{GHz}$ using a metallic photonic crystal (MPC) resonator have been demonstrated at MIT, which operated with a $\mathrm{TE}_{0,4}$-like mode [8]. In 2005, E.I. Smirnova developed a $17-\mathrm{GHz}$ high gradient accelerating cavity with a $\mathrm{PBG}$, whose acceleration gradient was up to $35 \mathrm{MV} / \mathrm{m}$ [9]. In 2006, the university of Utah developed backward-wave oscillators

This manuscript is submitted on 22nd, March, 2019. This work is sponsored by the National Natural Science Foundation of China under Contracts 61531002, 61861130367, and NSAF U1830201, and is also sponsored in part by Newton Advanced Fellowship NAF $\backslash$ R $1 \backslash 180121$ from Royal Society, United Kingdom. (Corresponding authors: Chao-Hai Du.)

Z. W. Zhang, C. H. Du, F. H. Li, J. F. Zhu and P. K. Liu are with the School of Electronics Engineering and Computer Science, Peking University Beijing 100871, China (e-mail: duchaohai@pku.edu.cn)

L. Zhang, W. He, H. Yin K. Ronald, A.D.R. Phelps, A.W. Cross are with the Department of Physics, SUPA, University of Strathclyde, Glasgow G4 0NG, United Kingdom. W. L. He is also with the College of Electronic Science and Technology, Shenzhen University, Shenzhen 518061, China. for use above 1-THz, which employ PC waveguide circuits for the slow-wave circuits [10]. In 2013, E.A. Nanni designed a 250-GHz gyrotron traveling wave tube using a PBG structure, and obtained a gain of more than $50 \mathrm{~dB}$ [1].

However, the previous resonant cavities have demanding requirements in terms of the magnitude of the magnetic field and none of them worked with a fourth-harmonic mode. In this paper, we focus on a square lattice of metallic rods as shown Fig. 1(a) and an MPC resonant cavity working at the fourth-harmonic which has been designed to suppress the mode competition. The gap configuration of the 2-D square lattice MPC was calculated using a Finite-Difference Frequency-Domain (FDFD) method along the irreducible Brillouin zone (IBZ) boundary, and the mode map of the circular waveguide enabled the design of the resonant cavity.

\section{THEORY OF PHTONIC CRYSTAL}

\section{A. Photonic Crystal}

A PC is a periodic arrangement of metal or dielectric structures. It usually consists of one-, two-, or threedimensional structures. The defining feature of a $\mathrm{PC}$ is the periodicity of the material along one or more axes. According to the lattice form, it can be divided into a triangular lattice PC or a square lattice PC.

\section{B. Master Equations of the 2-D PC}

As the relative magnetic permeability $\mu_{r}(\boldsymbol{r})$ is very close to unity, and there are no sources, we can set the free charge $\rho$ $=0$, current densities $\boldsymbol{J}=0$ and $\boldsymbol{B}=\mu_{0} \boldsymbol{H}$. For simplicity, the materials are linear and lossless and the field pattern varies harmonically with time. Since the system is homogeneous along the Z-direction the 2-D MCP structure, the master equations derived from Maxwell's equations are given by

$$
\left\{\begin{array}{l}
-\left(\frac{\partial H_{z}^{2}}{\partial^{2} x}+\frac{\partial H_{z}^{2}}{\partial^{2} y}\right)=\left(\frac{\omega}{c}\right)^{2} H_{z}(T E) \\
-\left(\frac{\partial E_{z}^{2}}{\partial^{2} x}+\frac{\partial E_{z}^{2}}{\partial^{2} y}\right)=\left(\frac{\omega}{c}\right)^{2} E_{z}(T M)
\end{array}\right.
$$

The master equations contain everything we need to know about the system along with the boundary conditions for metallic rods as specified below

$$
\left\{\begin{array}{l}
\left.\frac{\partial H_{z}}{\partial n}\right|_{S}=0(T E) \\
\left.E_{z}\right|_{s}=0(T \mathrm{M})
\end{array}\right.
$$

\section{Square Lattice and Irreducible Brillouin Zone}

The cross section of the square lattice is shown in Fig. 1(a) and the center blue square is a unit cell. Fig. 1(b) shows the reciprocal space of the square lattice, where the red triangle is an IBZ. The variation of the wave vector $k$ of the square lattice along the IBZ is shown in Table I. Due to its translational 


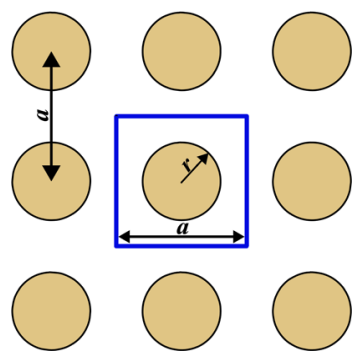

(a)

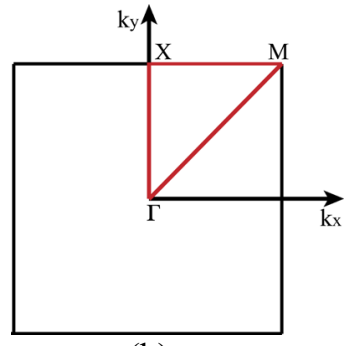

(b)
Fig. 1. (a) Structure of square lattice with rod radius $r$ and lattice constant $a$. (b) Reciprocal space of square lattice, Where $\Gamma, \mathrm{M}$ and $\mathrm{X}$ are symmetry points. Triangle determined by red lines is Irreducible Brillouin Zone.

TABLE I.

WAVE VECTOR OF IRREDUCIBLE BRILLOUIN BOUNDARY

\begin{tabular}{|c|c|c|}
\hline Direction & \multicolumn{2}{|c|}{ Wave vector k } \\
\hline$\Gamma \mathrm{X}$ & $(0,0)$ & $(0, \pi / \mathrm{a})$ \\
$\mathrm{XM}$ & $(0, \pi / \mathrm{a})$ & $(\pi / \mathrm{a}, \pi / \mathrm{a})$ \\
$\mathrm{M} \Gamma$ & $(\pi / \mathrm{a}, \pi / \mathrm{a})$ & $(0,0)$ \\
\hline
\end{tabular}

symmetry, the unit cell should satisfy the square lattice periodic boundary conditions in the periodic direction.

$$
\left\{\begin{array}{l}
\Psi(x+a, y)=e^{-j k_{x} a} \Psi(x, y) \\
\Psi(x, y+a)=e^{-j k_{y} a} \Psi(x, y)
\end{array}\right.
$$

Where $\Psi$ is the electric field or magnetic field in the unit cell, and $k_{x}$ and $k_{y}$ are the wave vector along the $\mathrm{x}$ and $\mathrm{y}$ direction separately.

\section{Dispersion Relations and Gap Map}

The dispersion relations describe the band structure of the PC. Using the FDFD method, the master equations of the square lattice MPC is calculated along the boundary of IBZ, and the dispersion relations can be obtained.

A plot of the locations of the PBG of a crystal, as one or more of the parameters of the crystal are varied, is what we call a gap map [7]. The gap map for the square lattice of metallic rods can be derived from dispersion relations as rod radius varies. Fig. 2 shows gap maps for the TE modes in square lattice as a function of ratio of rod radius $r$ to lattice constant $a$, which agrees with the outcome in [11].

\section{DESIGN OF RESONANT CAVITY}

In order to design a resonant cavity using $\mathrm{PC}$, it is necessary to introduce defects by removing several metallic rods. The modes whose frequency is inside the PBG can be confined in defects as a certain high-order mode, and the modes outside the band gap are scattered in the lateral direction of the waveguide and thus are suppressed. Fig. 3 shows the structure of a MPC cavity with 21 rods removed from the center.

The relationship between the modes of a circular waveguide and the cut-off radius is given by:

$$
R=\frac{c p_{m n}^{\prime}}{\omega}=\frac{p_{m n}^{\prime} a}{f_{\text {Norm }} \cdot 2 \pi}
$$

where $p_{m n}^{\prime}$ is the $n$-th root of the derivative of the $m$-order Bessel function, and the normalized frequency $f_{\text {Norm }}=f \cdot a / c$.

From Fig. 3, the equivalent operation radius $R$ of the

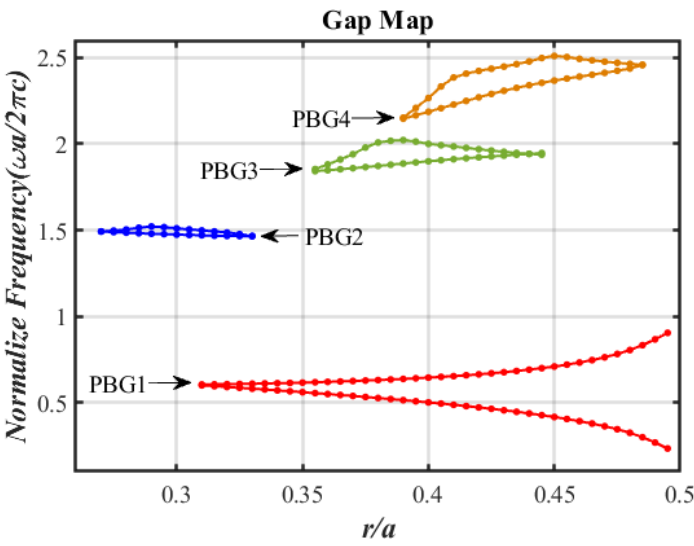

Fig. 2. Gap map of square lattice MPC for TE polarization. The part enclosed by the same color is the PBG. The four PBGs are plotted with different color and labeled as PBG1, PBG2, PBG3 and PBG4.

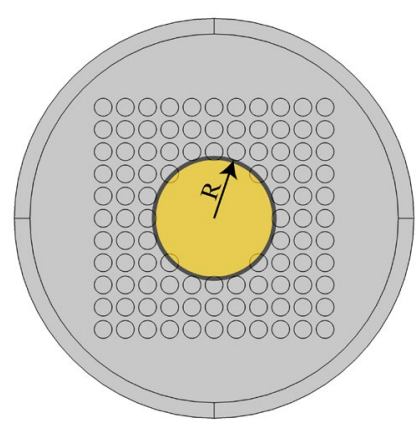

Fig. 3. Square lattice cavity with 21 rods removed from the center. R is the radius of equivalent circular waveguide. The lattice constant of the lattice is $a$ and the radius of $\operatorname{rod}$ is $r$.

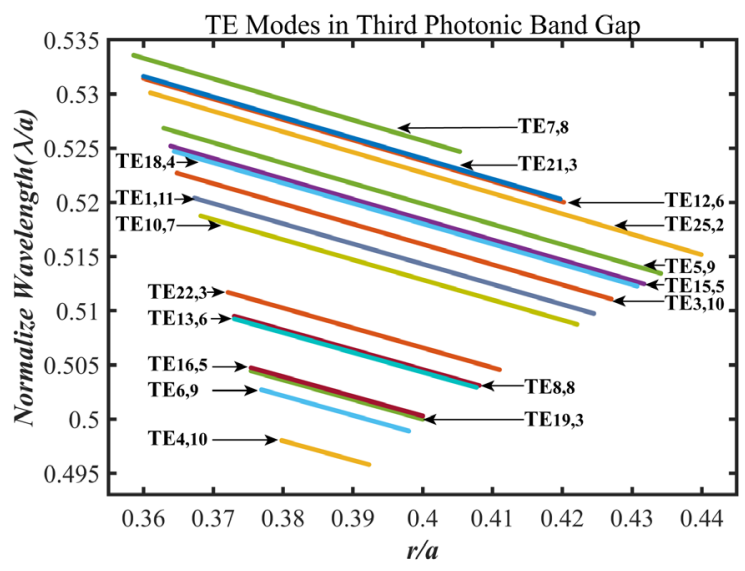

Fig. 4. Mode map of PBG3. There are 17 modes plotted, the $\mathrm{TE}_{4,10}$ mode is located at the bottom of figure.

equivalent circular waveguide (ECW) is $R=\sqrt{10} a-r$, therefore, we have

$$
\lambda_{\text {Norm }}=\frac{\lambda}{a}=\frac{(\sqrt{10} a-r) \cdot 2 \pi}{p_{m n}^{\prime} \cdot a}=\frac{2 \pi}{p_{m n}^{\prime}}\left(\sqrt{10}-\frac{r}{a}\right)
$$

where the normalized wavelength $\lambda_{\text {Norm }}=1 / f_{\text {Norm }}$.

The mode map of the circular waveguide from the gap map can be obtained using (5) and is shown in Fig. 4.

Due to the fact that the cavity boundary where the rods are removed is not perfectly circular, the field distribution in the cavity is slightly different from the field in a circular 


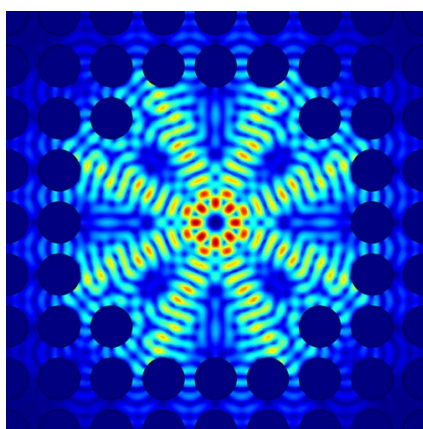

(a)

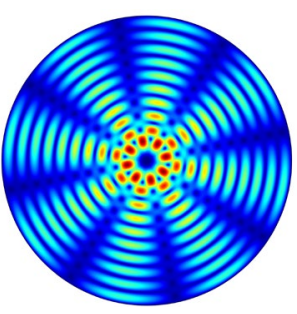

(b)
Fig. 5. (a). Mode pattern of $\mathrm{TE}_{4,10}$-like eigenmode in the MPC cavity at $1000.8 \mathrm{GHz}$. (b). Mode pattern of the $\mathrm{TE}_{4,10}$ eigenmode in the equivalent cylindrical cavity at $1010.6 \mathrm{GHz}$.

TABLE II

The Comparison of Circular Waveguide Mode Between The PHOTONIC CRYSTAL RESONATOR AND THE EQUIVALENT CYLINDRICAL WAVEGUIDE RESONATOR

\begin{tabular}{|l|c|c|c|c|c|}
\hline $\begin{array}{c}\text { Mode } \\
\text { in } \\
\text { ECW }\end{array}$ & $\begin{array}{c}\text { Frequency } \\
\text { (GHz) }\end{array}$ & $\begin{array}{c}\text { Ohmic } \\
\text { Q } \\
\text { factor }\end{array}$ & $\begin{array}{c}\text { Mode } \\
\text { in } \\
\text { MPC }\end{array}$ & $\begin{array}{c}\text { Frequency } \\
\text { (GHz) }\end{array}$ & $\begin{array}{c}\text { Ohmic } \\
\text { Q } \\
\text { factor }\end{array}$ \\
\hline $\mathrm{TE}_{16,5}$ & 999.3 & 15412 & & & \\
\hline $\mathrm{TE}_{6,9}$ & 1002.3 & 17600 & & & \\
\hline $\mathrm{TE}_{4,10}$ & 1010.6 & 17586 & $\mathrm{TE}_{4,10}$ & 1000.8 & 8989.6 \\
\hline $\mathrm{TE}_{11,7}$ & 1012.4 & 17132 & & & \\
\hline $\mathrm{TE}_{2,11}$ & 1015.6 & 18183 & & & \\
\hline $\mathrm{TE}_{0,11}$ & 1017.2 & 16843 & & & \\
\hline
\end{tabular}

waveguide. But the higher the radial index is, the better similarity they have. We choose PBG3 as operating band gap because there are fourth-harmonic mode in the gap, which has a higher radial index as shown in Fig. 4 of the plot of the circular waveguide mode in the PBG3 gap map, where $\lambda_{\text {Norm }}$ is the normalized wavelength of $\mathrm{TE}_{\mathrm{m}, \mathrm{n}}$ mode contained in the PBG cavity. Fig. 4 can be used to enable an informed choice for the dimensions of the lattice and the operation mode [12].

According to Fig. 4, we select the $\mathrm{TE}_{4,10}$ mode as the operating mode, which is at the bottom of Fig. 4. and far from the upper modes. The $\mathrm{TE}_{4,10}$ mode works in the range of 0.38 $<r / a<0.392$ and the normalized wavelength range is 0.4958 $<\lambda_{\text {Norm }}<0.498$. However, the calculation result of the PBG is obtained in the case of an ideal PC without defects. When the defects are introduced, the band gap may change slightly, and the mode map within the band gap is changed accordingly. The final parameters were chosen as $r / a=0.392$ and $a=0.6 \mathrm{~mm}$.

The structure was simulated using the Finite Element Method and the mode pattern of the $\mathrm{TE}_{4,10}$-like mode at 1.0008-THz is shown in Fig. 5(a). Most of the electromagnetic energy is distributed in the center of the cavity, which means the mode is localized in the defects. $\mathrm{A} \mathrm{TE}_{4,10}$ mode in the ECW with equivalent radius $R$ is simulated and plotted in Fig. 5 (b). Since the inner edge of MPC cavity is not a perfect circle, the deformation appears in Fig. 5.(a). Along the radial direction, the farther away from the axis, the more severe the distortion. But the mode distribution in the center of the cavity is exactly similar with that of the ECW, which is in good agreement with the theoretical analysis.

The comparison of the circular waveguide mode between the MPC resonator and ECW resonator is shown in Table II. Due to limitations in computer performance, only simulations of the $\mathrm{TE}_{\mathrm{m}, \mathrm{n}, 0}$ mode have been made here. It can be seen from Table II that PC can effectively suppress the competition of the circular-waveguide-like mode.

\section{SUMMERY}

In this paper, the master equations of the 2-D PC are derived. The gap map of the square lattice for TE type modes was calculated by solving the master equations using FDFD method. A square lattice resonator was designed by removing 21 metallic rods from the center. The mode map of the ECW is plotted in the PBG3. A MPC resonant cavity working in the $\mathrm{TE}_{4,10}$-like mode was designed and simulated at 1000.8-GHz. The mode density and the quality factor of the designed PBG cavity is compared with those of a ECW for neighboring modes around the operating frequency. As in [13], the cavity discussed above can be used to reduce the magnetic field strength and to suppress the mode competition for $\mathrm{THz}$ gyrotrons.

\section{ACKNOWLEDGEMENT}

The authors would like to thank the Royal Society of London, UK, for providing the Newton Advanced Fellowships (NAF\R1 \180121) for Professor Chao-Hai Du.

\section{REFERENCES}

[1] Nanni, Emilio Alessandro. A $250 \mathrm{GHz}$ photonic band gap gyrotron amplifier. Diss. Massachusetts Institute of Technology, 2013.

[2] Bailey, Aimee G., et al. "Photonic band gap structures for millimeterwave traveling wave tubes." Terahertz and Gigahertz Electronics and Photonics V. Vol. 6120. International Society for Optics and Photonics, 2006.

[3] Liu Pu-Kun, Du Chao-Hai. "Review of the Gyrotron Traveling WaveTube Amplifier Development." Journal of Microwaves, 2004, 76(2):489-540. (in Chinese)

[4] Liu Chang, Luo Yao-Tian Tang Chang-Jian, Liu Pu-Kun. "Electromagnetic mode analysis on the cold characteristics of photonic-band-gap resonant cavity loaded in gyrotron. " ACTA PHYSICA SINICA, 2009, 58(12):8174-8179. (in Chinese)

[5] John, Sajeev. "Strong localization of photons in certain disordered dielectric superlattices." Physical review letters 58.23 (1987): 2486.

[6] Yablonovitch, Eli. "Inhibited spontaneous emission in solid-state physics and electronics." Physical review letters 58.20 (1987): 2059.

[7] Winn, J. N., et al. "Photonic Crystals: Molding the flow of light." Princeton University Press, Princeton, NJ 6 (1995): 2059-2062.

[8] Sirigiri, Jagadishwar Rao, et al. "Photonic-band-gap resonator gyrotron." Physical Review Letters 86.24 (2001): 5628.

[9] Smirnova, Evgenya I., et al. "Demonstration of a 17-GHz, highgradient accelerator with a photonic-band-gap structure." Physical review letters 95.7 (2005): 074801.

[10] Vela, G. O. , et al. "Terahertz Backward-wave Oscillators with Photonic Crystal Waveguides." IEEE International Vacuum Electronics Conference IEEE, 2006.

[11] Smirnova, E. I., et al. "Simulation of photonic band gaps in metal rod lattices for microwave applications." Journal of Applied Physics 91.3 (2002): 960-968.

[12] Zhang, Yanyan, et al. "Analysis of the Photonic Bandgaps for Gyrotron Devices." IEEE Transactions on Plasma Science 43.4 (2015): 10181023.

[13] Du, Chao-Hai, et al. "Theoretical study of a fourth-harmonic 400-GHz gyrotron backward-wave oscillator." IEEE Transactions on Electron Devices 62.1 (2015): 207-212. 